06,19

\title{
Флексокалорический эффект в тонких пластинах титаната бария и титаната стронция
}

\author{
(C) A.С. Старков ${ }^{1}$, И.А. Старков ${ }^{1,2}$ \\ ${ }^{1}$ Национальный исследовательский университет информационных технологий, механики и оптики, \\ Санкт-Петербург, Россия. \\ ${ }^{2}$ Санкт-Петербургский государственный электротехнический университет „ЛЭТИ“ им. В.И. Ульянова (Ленина), \\ Санкт-Петербург, Россия. \\ E-mail: ferroelectrics@ya.ru
}

Поступила в Редакцию 24 июня 2019 г.

В окончательной редакции 24 июня 2019 г.

Принята к публикации 16 июля 2019 г.

На основе подхода Лява-Кирхгофа исследован флексоэлектрический эффект в тонкой пластинке сегнетоэлектрика, обладающего кубической симметрией. Электрическое и упругое поля в сегнетоэлектрике описываются в рамках термодинамического потенциала Ландау-Гинзбурга. Учтено влияние неоднородности распределения поляризации в пластинке. Найденные значения для изгиба пластинки, вызванного приложением электрического поля, дают возможность вычислить зависимость изменения энтропии от температуры в пластинках титаната бария и титаната стронция (флексокалорический эффект).

Ключевые слова: сегнетоэлектрики, тонкая пластинка, флексокалорический эффект, флексоэлектрический эффект, мультикалорический эффект, упругое смещение.

DOI: $10.21883 /$ FTT.2019.12.48586.527

\section{1. Введение}

Эффекты, связанные с изгибом твердого тела, носят приставку флексо, которая происходит от латинского глагола flex - сгибать, изгибать. Мы ограничимся описанием только флексоэлектрического эффекта (FEE) и тепловых эффектов, связанных с ним. Этот эффект был предсказан первым из флексоэффектов [1] и заключается в возникновении поляризации при наличии градиента деформации. Обратный эффект состоит в появлении неоднородной деформации, например, изгиба, обусловленного существованием поляризации. Помимо неоднородной деформации FEE может возникать в нематических жидких кристаллах при пространственной модуляции директора, указывающего ориентацию молекул. Первая феноменологическая теория FEE была создана в 1963 г. Ш.М. Коганом [2]. В этой же работе появились оценки флексоэлектрических коэффициентов. Экспериментальное наблюдение обратного FEE относится к 1968 г. [3], когда было измерено искривление тонкой пластинки титаната бария $\mathrm{BaTiO}_{3}$, вызванное поляризацией. Сам термин „флексоэлектрический эффект“ появился в работе [4], а до того этот эффект назывался „нелокальным пьезоэлектрическим эффектом“ [5].

Зависимость флексоэлектрической части энергии от температуры $T$ приводит к существованию флексокалорического эффекта (FCE). Этот эффект по терминологии [6] следует отнести к третичным калорическим эффектам, которые вызываются градиентами электрического, магнитного или упругого поля. Термин FCE и теория, описывающая этот эффект, впервые появились в работе [7]. При FCE происходит изменение температуры или энтропии образца, которое обусловлено наличием неоднородной деформации. Согласно теоретическим расчетам на основе модели Ландау-Гинзбурга [7] при градиенте деформации $1 \mathrm{~m}^{-1}$ для $\mathrm{PMN}$ керамики изменение температуры при FCE зависит от температуры $T$ и меняется от $5 \mu \mathrm{K}$ при $T=240 \mathrm{~K}$ до $-6 \mu \mathrm{K}$ при $T=299 \mathrm{~K}$. Расчеты на основе первых принципов подтвердили существование FCE в BST [2]. Величина эффекта составила $1.5 \mathrm{~K}$ при температуре $T=289 \mathrm{~K}$ и градиенте деформации $1.5 \mu \mathrm{m}^{-1}$, т. е. увеличение градиента деформации на шесть порядков приводит примерно к такому же увеличению FCE. Наибольшее значение FCE для BST керамики, полученное в результате расчетов, составляет $60 \mathrm{~K}$. Но достигается оно в слое толщиной порядка $1 \mathrm{~nm}$ [9]. Величина FCE в данном случае определяется огромными значениями напряжения рассогласования $\left(10^{6}-10^{7} \mathrm{~m}^{-1}\right)$, существующего на границах двух кристаллов с разными постоянными атомных решеток.

В последние годы интерес к флексоэффектам значительно вырос. Вызвано это следующими обстоятельствами. Экспериментально полученные значения флексокоэффициентов [10] оказались на три порядка больше, чем предсказываемые теорией [2]. Возникли новые области применения флексоэффектов [11]. К перечисленным в этой работе практическим приложениям также следует добавить возможность создания наногенераторов [12] и охлаждающих устройств [12] на FEE. Кроме того, флексоэффекты позволили описать ряд тонких физических явлений, таких как изменение температуры фазового перехода, пьезоэлектрический отклик и пространственное 

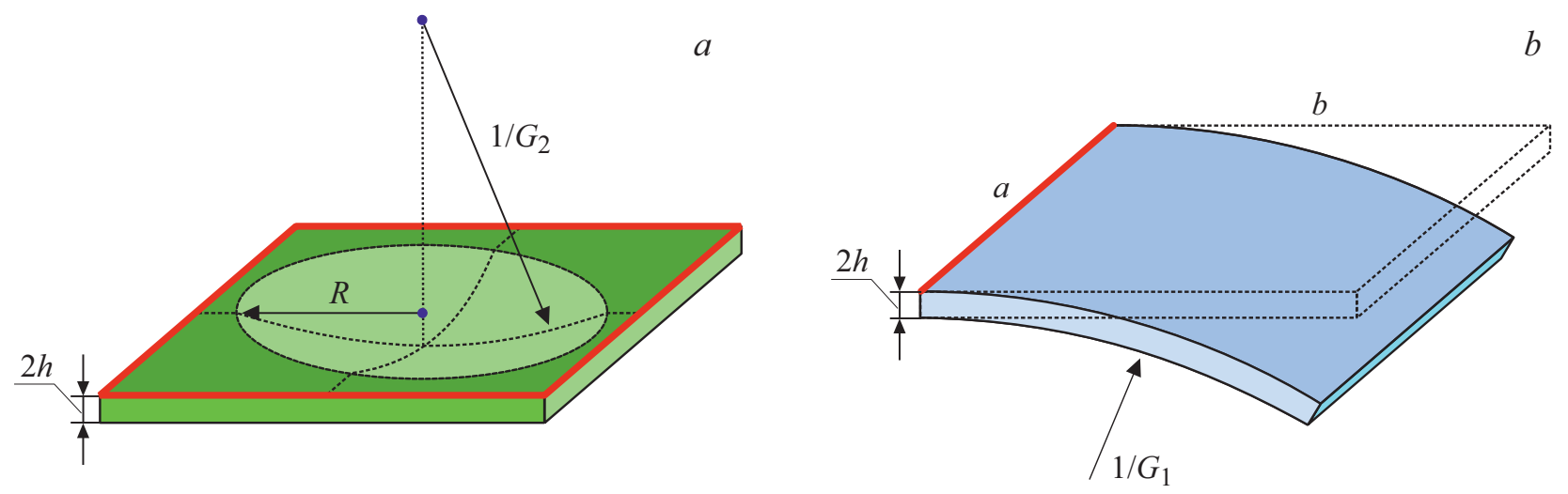

Рис. 1. Сегнетоэлектрическая пластинка (a) круглой формы закрепленная по внешнему периметру, и $(b)$ прямоугольной формы, закрепленная по одному краю.

распределение поляризации [14]. Одним из основных достоинств флексоэффектов является их усиление при уменьшении размеров образца. Например, для пленок титаната бария $\mathrm{BaTiO}_{3}$ толщиной $20 \mathrm{~nm}$ величина FCE совпадает с величиной электрокалорического эффекта, а при меньших толщинах превосходит ее. Ввиду этого, изучение флексоэффектов для микро- и наноразмерных объектов представляет несомненный научный интерес. В настоящей работе исследуются флексоэлектрический и флексокалорический эффекты в тонкой пластинке сегнетоэлектрика, обладающего кубической симметрией. Описание поляризации и упругих смещений в пластинке основывается на термодинамическом потенциале Ландау-Гинзбурга, в который добавлены слагаемые, учитывающие FEE [14]. Ранее флексоэлектрическая пластинка рассматривалась в [15], где автор использовал подход Лява-Кирхгофа для описания тонких пластин [16]. В настоящей работе при описании FEE применяется тот же подход, но дополнительно учитывается неоднородность распределения поляризации по пластине. Для простоты изложения не учитывается электрострикционный эффект. Для включения его в нижеизложенную схему нахождения векторов поляризации и смещений достаточно включить дополнительные слагаемые в разложение этих векторов. Можно показать, что с точностью до малых слагаемых электрострикционный эффект для тонких пластин ведет просто к появлению равномерной деформации, т.е. этот эффект и FEE можно рассматривать независимо друг от друга. Данный вывод подтверждается и экспериментальными результатами для титанатов стронция [17] и бария [18].

\section{2. Флексоэлектрическая пластинка}

\section{1. Обратный флексоэлектрический эффект в пластинке}

В декартовой системе координат рассмотрим сегнетоэлектрическую пластинку толщиной $2 h$. В качестве примера будем исследовать либо круглую пластинку радиуса $R$, либо прямоугольную со сторонами $a$ и $b$ (см. рис. 1). В соответствии с условиями эксперимента, будем считать круглую пластинку закрепленной по внешнему периметру $[18,19]$. Прямоугольную пластину считаем закрепленной по одному краю, а оставшиеся три края считаем свободными [17]. Толщина пластинки является малой по сравнению с радиусом $R$ или ее шириной $a$ и длиной $b, h \ll R, h \ll a, h \ll b$. К сторонам пластинки прикладывается разность потенциалов $U$, которая создает однородное электрическое поле с напряженностью $E=U /(2 h)$. При этом происходит изгиб пластинки, вызванный обратным флексоэлектрическим эффектом. Отыскание явных формул для этого изгиба является основной целью настоящей работы. На границе флексоэлектрической пластинки возникает поверхностный слой, толщина которого составляет несколько нанометров [13]. Влиянием этого слоя можно пренебречь, если рассматривать пластинки толщиной $2 h>100 \mathrm{~nm}$. Будем предполагать, что имеется только одна вертикальная компонента у электрического поля $E_{3}$ и одна компонента у поляризации $P_{3}$. При этих предположениях и кубической симметрии материала свободная энергия пластинки имеет вид [14].

$$
\begin{aligned}
& F=\frac{c_{11}}{2}\left(u_{11}^{2}+u_{22}^{2}+u_{33}^{2}\right)+c_{12}\left(u_{11} u_{22}+u_{33} u_{22}+u_{11} u_{33}\right) \\
& +2 c_{44}\left(u_{23}^{2}+u_{13}^{2}+u_{12}^{2}\right)+\frac{f_{11}}{2}\left(u_{33,3} P_{3}-u_{33} P_{3,3}\right) \\
& +\frac{f_{12}}{2}\left[\left(u_{11,3}+u_{22,3}\right) P_{3}-\left(u_{11}+u_{22}\right) P_{3,3}\right] \\
& +\frac{f_{44}}{2}\left(u_{23,2}+u_{13,1}\right) P_{3}+a_{1} \frac{P_{3}^{2}}{2}+a_{2} \frac{P_{3}^{4}}{4}+a_{3} \frac{P_{3}^{6}}{6}-E_{3} P_{3} .
\end{aligned}
$$

Здесь $c_{i j}$ - упругие модули, $f_{i j}-$ флексоэлектрические коэффициенты, $u_{i j}=\left(u_{i, j}+u_{j, i}\right) / 2-$ компоненты тензора деформации, $u_{i}$ - компоненты вектора смещения, $a_{i}(i=1,2,3$,$) - коэффициенты Гинзбурга-$ Ландау. Индекс после запятой означает дифференцирование по соответствующей переменной. Свободная 
энергия (1) не является положительно определенным функционалом. Из-за этого выражение (1) нельзя использовать для пластин толщиной несколько нанометров. Для обеспечения положительной определенности в нее следует добавить слагаемые, пропорциональные градиентам поляризации и деформации. Именно эти слагаемые ответственны за возникновение пограничных слоев на границах раздела различных сред. Можно показать, что для пластин толщиной несколько микрон этими слагаемыми можно пренебречь и рассматривать функционал (1), несмотря на его математическую некорректность. Пластинку считаем свободной, т. е. при $z= \pm h$ должны выполняться граничные условия $[17,20]$, которые обобщают условия обращения в ноль нормальных составляющих тензора напряжений в классической теории упругости. В соответствии с подходом ЛяваКирхгофа, при малых толщинах пластинки в разложении искомых величин по степеням $z$ можно ограничиться только первыми слагаемыми, а именно, искать упругие смещения и поляризацию

$$
\begin{aligned}
& u_{3}=w+w_{2} \frac{z^{2}}{2}, \quad u_{1}=-z w_{, 1}, \\
& u_{2}=-z w_{, 2}, \quad P_{3}=P+P_{2} \frac{z^{2}}{2},
\end{aligned}
$$

где $w(x, y), w_{2}(x, y), P(x, y), P_{2}(x, y)-$ новые неизвестные функции, которые могут зависеть только от продольных координат. Выражения для смещений $u_{1}$ и $u_{2}$ в (2) получены стандартным образом при интегрировании двух уравнений, полученных при варьировании (1) и учете граничных условий. Они совпадают с хорошо известными выражениями в теории упругости [16] и основаны на равенствах $u_{23}=u_{13}=0$. Аналогичным образом получаем выражение для коэффициента $w_{2}$, который имеет вид

$$
w_{2}=\frac{c_{12}}{c_{11}} \Delta w-\frac{f_{11}}{c_{11}} P_{2} .
$$

Здесь $\Delta$ есть двумерный оператор Лапласа. В отличие от обычной теории упругости в правой части (3) имеется дополнительное слагаемое, определяемое градиентом поляризации. Из уравнения отсутствия зарядов $\operatorname{div}\left(\varepsilon_{0} E_{3}+P_{3}\right)=0$ получаем, что $P_{2}$ не зависит от $x$ и $y$, т.е. является постоянной. Напомним, что $\varepsilon_{0}$ есть электрическая постоянная (диэлектрическая проницаемость вакуума). Подставим разложения (2), (3) в (1) и усредним полученное выражение по координате $z$ [16]

$$
\bar{F}=F_{\text {elast }}+F_{\delta, \text { elast }}+F_{\text {coupl }}+F_{\mathrm{LG}}+F_{\delta, \mathrm{LG}} .
$$

Черта над свободной энергией означает усреднение по пластине $\bar{F}=\int_{-h}^{h} F \mathrm{~d} z /(2 h)$. Здесь введены обозначения: $F_{\text {elast }}$ для обычной упругой энергии пластинки,

$$
\begin{aligned}
F_{\text {elast }}= & {\left[\frac{c_{11}^{2}-c_{12}^{2}}{2 c_{11}}(\Delta w)^{2}\right.} \\
& \left.+\left(c_{12}-c_{11}\right) w_{, 11} w_{, 22}+2 c_{44} w_{, 12}^{2}\right] \frac{h^{2}}{3},
\end{aligned}
$$

$F_{\delta \text {,elast }}$ для поправки к упругой энергии пластинки, вызванной наличием FEE,

$$
F_{\delta, \text { elast }}=\frac{f_{11}^{2} h^{2}}{3 c_{11}} P_{2}^{2},
$$

$F_{\text {coupl }}$ для энергии взаимодействия электрического и упругого полей,

$$
F_{\text {coupl }}=\left(f_{11} \frac{c_{12}}{c_{11}}-f_{12}\right)\left(P-\frac{h^{2}}{6} P_{2}\right) \Delta w,
$$

$F_{\mathrm{LG}}$ для стандартного термодинамического потенциала Ландау-Гинзбурга для сегнетоэлектрика,

$$
F_{\mathrm{LG}}=a_{1} \frac{P^{2}}{2}+a_{2} \frac{P^{4}}{4}+a_{3} \frac{P^{6}}{6}-E P,
$$

$F_{\delta \mathrm{LG}}$ для поправки к потенциалу $F_{\mathrm{LG}}$

$$
F_{\delta \mathrm{LG}}=\left(a_{1} P+a_{2} P^{3}+a_{3} P^{5}\right) \frac{P_{2} h^{2}}{6}-E P_{2} \frac{h^{2}}{3} .
$$

Варьирование по $P$ и $P_{2}$ приводит к уравнениям

$$
\begin{aligned}
E= & a_{1} P+a_{2} P^{3}+a_{3} P^{5}+\left(a_{1}+3 a_{2} P^{2}+5 a_{3} P^{4}\right) \frac{P_{2} h^{2}}{6} \\
& +\left(f_{11} \frac{c_{12}}{c_{11}}-f_{12}\right) \Delta w, \\
& {\left[-\frac{f_{11}}{2}\left(\frac{c_{12}}{c_{11}} \Delta_{2} w-\frac{f_{11}}{c_{11}} P_{2}\right)-\frac{c_{12}}{c_{11}} w f_{11}\right] \frac{h^{2}}{3} } \\
- & f_{12} \frac{h^{2}}{12} \Delta w-E \frac{h^{2}}{3}+\left(a_{1} P+a_{2} P^{3}+a_{3} P^{5}\right) \frac{h^{2}}{6} \\
& -\frac{f_{11}}{2} w_{2} \frac{h^{2}}{6}-\frac{f_{11}^{2}}{2 c_{11}}\left(P-\frac{h^{2}}{6} P_{2}\right)=0 .
\end{aligned}
$$

Варьирование (4) по $w$, так же как и в обычной теории упругости, ведет к бигармоническому уравнению

$$
\Delta^{2} w=0 .
$$

Отсюда следует, что наличие флексоэлектрической части свободной энергии сказывается только на граничных условиях на боковых сторонах пластины, вызванных присутствием $F_{\text {coupl. }}$ Решение уравнения (11) с указанными в начале раздела граничными условиями для прямоугольной $\left(w_{1}\right)$ и круглой $\left(w_{2}\right)$ пластинок имеет вид

$$
\begin{gathered}
w_{1}=-\frac{G_{1}}{2} x^{2}, \quad w_{2}=\frac{G_{2}}{2}\left(R^{2}-x^{2}-y^{2}\right), \\
G_{1}=2 G_{2}, \quad G_{1}=\frac{3\left(f_{11} c_{12}-f_{12} c_{11}\right)}{8 h^{2} c_{44}\left(c_{12}+c_{44}\right)}\left(P-\frac{h^{2}}{6} P_{2}\right) .
\end{gathered}
$$

Постоянные $G_{1,2}$ есть кривизны при одномерной (прямоугольная пластина) и двумерной (круглая пластина) деформациях. Радиус кривизны для пластинки при флексоэлектрической деформации оказывается в 2 раза больше 
радиуса кривизны круглой пластинки. Справедливость выведенного соотношения подтверждается экспериментом $[17,19]$. Нетрудно проверить, что для прямоугольной пластинки, зажатой с двух противоположных сторон, радиус кривизны будет в 4 раза меньше $G_{1}$. Отметим, что полученное выражение для флексоэлектрического изгиба пластины не содержит коэффициента $f_{44}$, который присутствует в работе [15]. При предположении об однородности электрического поля, имеющего только одну компоненту, этот коэффициент не входит в систему уравнений, описывающих поведение пластинки [14].

\section{2. Флексокалорический эффект в пластинке}

Перейдем к исследованию тепловых явлений в пластинке. Наиболее просто находится энтропия $S=-\partial F / \partial T$. Но для свободной энергии вида (1) или даже ее упрощенного варианта (4) выделить флексокалорический эффект в мультикалорическом чрезвычайно трудно [21]. Ввиду этого ограничимся только качественной оценкой данного эффекта. Для этого выделим в усредненной свободной энергии (4) главную часть, отвечающую флексоэлектрической энергии

$$
F_{\mathrm{FEE}}=\frac{\left(c_{11}^{2}-c_{12}^{2}\right) h^{2}}{6 c_{11}}(\Delta w)^{2}+\left(f_{11} \frac{c_{12}}{c_{11}}-f_{12}\right) P \Delta w
$$

В выражении (13) осталась только чисто механическая (первое слагаемое) и флексоэлектрическая части энергии. При подстановке (12) в (13) и отбрасывании слагаемого, пропорционального поляризации $P_{2}$, находим $F_{\mathrm{FEE}}=-G_{1}^{2}\left(c_{11}^{2}-c_{12}^{2}\right) h^{2} /\left(6 c_{11}\right)$. Пренебрегая температурной зависимостью упругих постоянных и толщины пластинки получаем для части энтропии, обусловленной флексоэлектрическим эффектом, следующее выражение

$$
S=\frac{\left(c_{11}^{2}-c_{12}^{2}\right) h^{2}}{3 c_{11}} G_{1} \frac{\partial G_{1}}{\partial T} .
$$

График зависимости изменения энтропии в кристалле $\mathrm{SrTiO}_{3}$ толщиной $2 h=165 \mu \mathrm{m}$ при подаче электрического поля $50 \mathrm{kV} / \mathrm{cm}$ представлен на рис. 2. Температурная зависимость кривизны $G_{1}$ взята из [17]. Используемая зависимость хорошо описывается законом Кюри-Весса $G_{1}=C /\left(T-T_{C}\right)$, где флексоэлектрический аналог постоянной Кюри $C=4.7 \mathrm{~K} / \mathrm{m}$, а температура Кюри $T_{C}=70 \mathrm{~K}$. Результаты интерполяции на основе данного закона для изменения энтропии отображены на рис. 2. Для титаната бария величина FCE должна быть существенно больше. Радиус прогиба для $\mathrm{BaTiO}_{3}$ оценивается в $20 \mathrm{~m}$ для электрического поля в $0.5 \mathrm{kV} / \mathrm{cm}$ при примерно таких же размерах кристалла, что и кристалл титаната стронция [18]. Поскольку данные для температурной зависимости величины прогиба для $\mathrm{BaTiO}_{3}$ в литературе не найдены, то для построения зависимости было взято одно экспериментальное значение $G_{1}$ при комнатной температуре. Для произвольной температуры

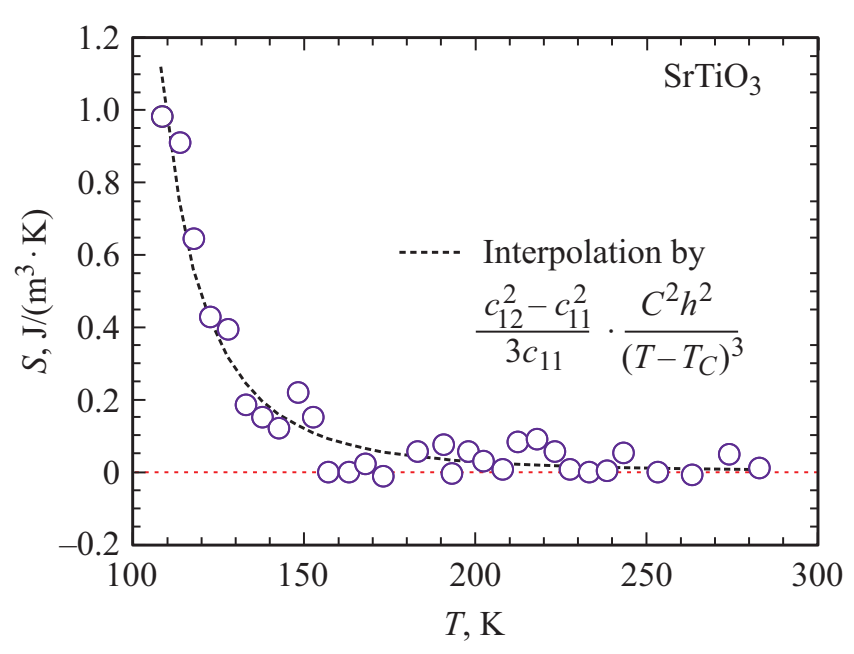

Рис. 2. Температурная зависимость изменения энтропии при флексокалорическом эффекте в кристалле $\mathrm{SrTiO}_{3}$.

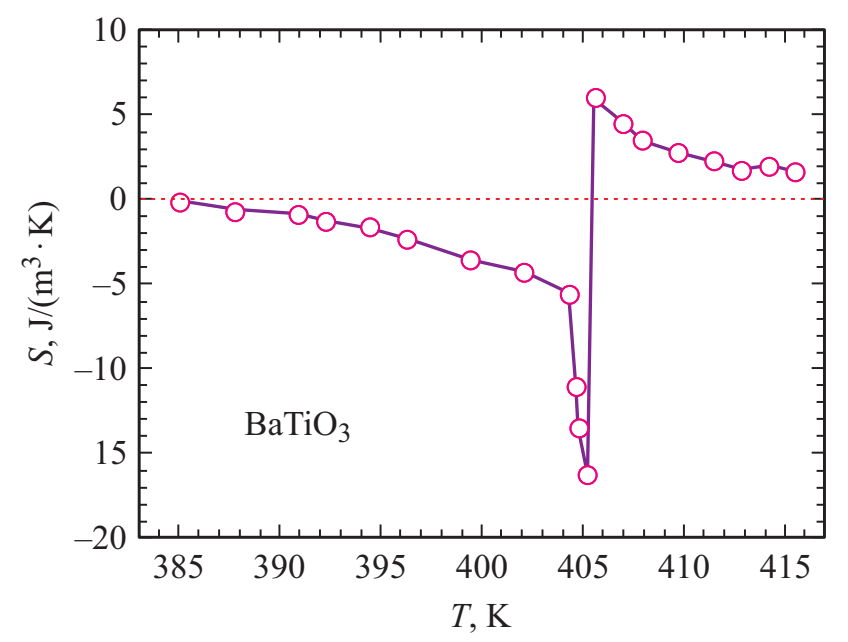

Рис. 3. Температурная зависимость изменения энтропии при флексокалорическом эффекте в кристалле $\mathrm{BaTiO}_{3}$.

использовалась пропорциональность $G_{1}$ и диэлектрической проницаемости $G_{1}(T) / G_{1}\left(T_{\mathrm{r}}\right) \sim \varepsilon(T) / \varepsilon\left(T_{\mathrm{r}}\right)$, где $T_{\mathrm{r}}$ - температура окружающей среды. Отсюда следует $\partial G_{1}(T) / \partial T \sim G_{1}\left(T_{\mathrm{r}}\right) / \varepsilon\left(T_{\mathrm{r}}\right) \cdot \partial \varepsilon(T) / \partial T$. По полученной зависимости $G_{1}(T)$ для титаната бария построен график зависимости FCE от температуры (рис. 3). При расчетах использовались упругие постоянные, приведеенные в [22].

\section{3. Заключение}

Из двух выведенных уравнений (10), (11) мы использовали только хорошо известное бигармоническое уравнение (11), так как для интерпретации его решений имеются надежные экспериментальные данные. Полученная формула для кривизны изгиба пластинки $G_{1}(12)$, вызванного электрическим полем, имеет та- 
кую же структуру, как выражения, полученные в [20], но несколько от них отличается. Применение подхода Лява-Кирхгофа позволило уточнить зависимость $G_{1}$ от поляризации и включить в формулу (12) дополнительное слагаемое. Наибольшая величина флексокалорического эффекта достигается при максимальном изгибе, который возникает в прямоугольной пластине с одной закрепленной стороной. Для круглых пластин эффект уменьшается в 2 раза, а для закрепленной с двух сторон прямоугольной пластины - в 4 раза. FEE и FCE сильно зависят от толщины пластинки. Из формул (10), (11) следует, что данная зависимость является достаточно сложной. В [3] на основе имеющихся экспериментальных данных утверждается, что зависимость от толщины можно аппроксимировать степенной с показателем -1.6. Принимая во внимание, что в знаменатель формулы (12) входит $h^{2}$, но помимо этой основной зависимости толщина $h$ имеется в уравнениях (10), (11), следует ожидать, что эмпирический закон $h^{-1.6}$ выполняется только до некоторой толщины $h_{\mathrm{th}}$, а при толщинах, меньших $h_{\mathrm{th}}$, происходит насыщения, и дальнейшего увеличения прогиба при уменьшении толщины пластинки не происходит. Для кристалла BST, согласно нашим оценкам, $h_{\text {th }} \sim 20-100 \mathrm{~nm}$.

Полученные величины флексокалорического эффекта являются весьма малыми и составляют менее $0.1 \%$ от достигнутых значений различных калорических эффектов [23]. Тем не менее, при уменьшении толщины пластинки на два порядка (до $160 \mathrm{~nm}$ ) следует ожидать, что величина FCE сравняется с величинами электро- и барокалорического эффектов. Значит, флексокалорический эффект, вызванный изгибом пластинки, существенно превосходит FCE, обусловленный неоднородностью электрического поля [7,8]. Это позволяет надеяться на использование флексоэлектрического эффекта в новом поколении твердотельных охладителей и генераторов.

\section{Финансирование}

Работа выполнена при финансовой поддержке Российского Научного Фонда (грант № 18-19-00512).

\section{Конфликт интересов}

Авторы заявляют об отсутствии потенциального конфликта интересов, требующего раскрытия в данной статье.

\section{Список литературы}

[1] В.С. Машкевич, К.Б. Толпыго. ЖЭТФ 32, 520 (1957).

[2] Ш.М. Коган. ФТТ 5, 2829 (1963).

[3] Э.В. Бурсиан, О.И. Зайковский. ФТТ 10, 1413 (1968).

[4] В.Л. Инденбом, Е.Б. Логинов, М.А. Осипов. Кристаллография 26, 1157 (1981).

[5] Э.В. Бурсиан, Н.Н. Трунов. ФТТ 16, 1187 (1974).
[6] J.F. Nye. Physical properties of crystals: their representation by tensors and matrices. Oxford University Press, N.Y. (1985). $352 \mathrm{p}$.

[7] А.С. Старков, И.А. Старков. ЖЭТФ 146, 297 (2014).

[8] S. Patel, A. Chauhan, J. Cuozzo, S. Lisenkov, I. Ponomareva, R. Vaish. Appl. Phys. Lett. 108, 162901 (2016).

[9] А.С. Старков, И.А. Старков. ФТТ 58, 1739 (2016).

[10] P. Zubko, G. Catalan, A. Buckley, P.R.L. Welche, J.F. Scott. Phys. Rev. Lett. 99, 167601 (2007).

[11] P. Zubko, G. Catalan, A.K. Tagantsev Annu. Rev. Mater. Res. 43, 387 (2013).

[12] Q. Deng, M. Kammoun, A. Erturk, P. Sharma. Int. J. Solids and Struct. 51, 3218 (2014).

[13] A.S. Starkov, I.A. Starkov. Int. J. Solids and Struct. 8265 (2016).

[14] E. A. Eliseev, A. N. Morozovska, M. D. Glinchuk, R. Blinc. Phys. Rev. B 79, 165433 (2009).

[15] А.С. Юрков. ФТТ 57, 450 (2015).

[16] Л.Д. Ландау, Е.М. Лифшиц. Теория упругости. Наука, М. (1987). 248c

[17] В.Г. Залесский, Е.В. Румянцева. ФТТ 56, 1301 (2014).

[18] Е.Д. Румянцева, В.Г. Залесский. ФТТ 58, 671 (2016).

[19] Е.Д. Румянцева, В.Г. Залесский. ПЖЭТФ 103, 792 (2016).

[20] А.С. Юрков. ПЖЭТФ 94, 455 (2011).

[21] I.A. Starkov, A.S. Starkov. Int. J. Solids and Struct. 100, 187 (2016).

[22] Z.G. Ban, S.P. Alpay. Phys. Rev. B. bf 67, 184104 (2003).

[23] И.Н. Флеров, Е.А. Михалева, М.В. Горев, А.В. Карташев. ФTT 57, 421 (2015).

Редактор Е.Ю. Флегонтова 